\title{
A Questionnaire-based Study of the Views of Schizophrenia Patients and Psychiatric Healthcare Professionals in Japan about the Side Effects of Clozapine
}

\author{
Ippei Takeuchi ${ }^{1,4}$, Manako Hanya ${ }^{4}$, Junji Uno ${ }^{1}$, Yuhei Amano ${ }^{2}$, Keiko Fukai ${ }^{3}$, Kiyoshi Fujita ${ }^{1}$, Hiroyuki Kamei ${ }^{4}$ \\ ${ }^{1}$ Department of Psychiatry, Okehazama Hospital, Toyoake, Aichi, ${ }^{2}$ Department of Psychiatry, Kakamigahara Hospital, Kakamigahara, Gifu, \\ ${ }^{3}$ Department of Psychiatry, Numazu Chuo Hospital, Numazu, Shizuoka, ${ }^{4}$ Office of Clinical Pharmacy Practice and Health Care Management \\ Faculty of Pharmacy, Meijo University, Nagoya, Japan
}

\begin{abstract}
Objective: It is well documented that clozapine treatment causes agranulocytosis, but it can also induce drowsiness, constipation, and hypersalivation; however, these symptoms are usually less severe. It has been reported that clozapine-treated patients with schizophrenia and psychiatric healthcare professionals consider different side effects to be important. The aim of this study was to assess current practice related to the side effects of clozapine in clozapine-treated patients with schizophrenia and psychiatric healthcare professionals in Japan.

Methods: Data were collected from January 2014 to August 2015 in Okehazama Hospital, Kakamigahara Hospital, and Numazu Chuo Hospital. Clozapine-treated patients with schizophrenia and psychiatric healthcare professionals (psychiatrists and pharmacists) were enrolled in this study.

Results: Of the 106 patients and 120 psychiatric healthcare professionals screened, 100 patients and 104 healthcare professionals were included in this study. We asked the patients what side effects caused them trouble and we asked psychiatric healthcare professionals what side effects caused them concern. The patients and psychiatrists held similarly positive views regarding the efficacy of clozapine. The healthcare professionals were concerned about agranulocytosis (92.4\%), blood routines (61.3\%). On the other hand, the patients experienced hypersalivation (76.0\%), sleepiness (51.0\%). A positive correlation $(R=0.696)$ was found between patient satisfaction and DAl-10 score.

Conclusion: Patients experienced more problems than healthcare professionals expected. However, usage experience of clozapine healthcare professionals tended to have similar results to patients. It is necessary that all healthcare professionals fully understand the efficacy and potential side effects of clozapine. This is very important for promoting clozapine treatment in Japan.
\end{abstract}

KEY WORDS: Clozapine; Refractory schizophrenia; Questionnaire; Clozapine treatment; Side effect.

\section{INTRODUCTION}

The primary treatment for schizophrenia is antipsychotic medication. However, antipsychotic agents are not very effective against refractory schizophrenia. ${ }^{1)}$ Fortunately, clozapine has been demonstrated to be effective against refractory schizophrenia. ${ }^{2)}$ Although it has a favorable side effects profile, $1 \%$ to $2 \%$ of patients who receive clozapine discontinue treatment due to agranulocytosis. ${ }^{3,4)}$ Its other side effects, such as hypersalivation,

\footnotetext{
Received: January 27, 2016 / Revised: March 12, 2016

Accepted: March 21, 2016

Address for correspondence: Ippei Takeuchi, M.Sharm Department of Psychiatry, Okehazama Hospital, Minamiyakata 3-879, Sakae-cho, Toyoake, Aichi, 470-1168, Japan

Tel: +81-562-97-1361, Fax: +81-562-97-8004

E-mail: 134331503@ccalumni.meijo-u.ac.jp
}

drowsiness, constipation, hypotension, weight gain, and tachycardia, are usually less severe, but they can have significant impacts on tolerability, medication adherence, and quality of life. ${ }^{5}$ Furthermore, whilst the initiation of clozapine treatment is associated with a significantly lower rate of psychiatric hospital admission, ${ }^{6}$ ) some patients find it difficult to continue with clozapine treatment. It was reported that whilst psychiatrists considered agranulocytosis, cardiac complications, and rebound psychosis to be the most important issues associated with clozapine treatment, they thought that patients did not consider these issues to be so problematic. ${ }^{7}$ Unfortunately, the latter study focused on psychiatrists' views and did not include any feedback from patients. ${ }^{7)}$ In another study, patient feedback was included ${ }^{8)}$ but side effects were assessed using the Liverpool University Neuroleptic Side Effect

(c) This is an Open-Access article distributed under the terms of the Creative Commons Attribution Non-Commercial License (http://creativecommons.org/licenses/by-nc/4.0) which permits unrestricted non-commercial use, distribution, and reproduction in any medium, provided the original work is properly cited. 
Rating Scale (LUNSERS) questionnaire, ${ }^{9)}$ which is a tool for evaluating Parkinson's disease. Therefore, we do not have any reliable patient feedback about clozapine treatment. At present, substantial delays in the initiation of clozapine treatment and the use of antipsychotic polypharmacy and high doses are common prior to clozapine treatment, despite these approaches running counter to current treatment guidelines. ${ }^{10)}$ In addition, clozapine is an unfamiliar treatment in Japan. Refractory schizophrenia is estimated to affect 20,000-50,000 patients in Japan, although the actual figure in May 2015 was 3,419 patients. There are many reasons for clinicians' reluctance to use clozapine, for example, the lack of experience of using the drug among medical professionals, a lack of information regarding the effects and side effects of the drug, and the low hospital availability of clozapine. Therefore, healthcare professionals might avoid clozapine treatment. Moreover, the difference in the perception of the side effects of clozapine between patients and psychiatrists is a potential cause of reduced adherence. Good adherence is associated with symptom stability and the prevention of relapses and rehospitalization. ${ }^{11)}$ It was reported that $76 \%$ of patients with schizophrenia relapse within 1 to 2 years of discontinuing their antipsychotic medication. ${ }^{12)}$ So, it is important to improve patient adherence. Medication adherence is also associated with treatment satisfaction scores. ${ }^{13)}$

We need to understand the factors involved in patient adherence and the differences between patients' and healthcare professionals' views about clozapine treatment, as such knowledge would enable us to optimize clozapine treatment methods.

Therefore, this study was designed to evaluate patient feedback and the views of healthcare professionals regarding clozapine treatment.

\section{METHODS}

\section{Subjects}

Prior to the study, we identified 106 patients and 120 healthcare professionals as potential subjects, and we obtained consent from 100 patients and 104 healthcare professionals (who were included in the study). The participating patients were treated as outpatients or inpatients at Okehazama Hospital, Kakamigahara Hospital, and Numazu Chuo Hospital between January 2014 and August 2015. The patients had received clozapine treatment minimum one month more. The healthcare professionals (physicians and pharmacists) all worked at psychiatric hospitals. The study was described to the subjects, and written informed consent was obtained from each of them. The study protocol was approved by the ethics committee at Okehazama Hospital Fujita Kokoro Care Center on January 7th 2014 (No. H25-13).

\section{Methods}

Pharmacists conducted the patient questionnaires, including Patient Questions (PQ) 1-6 (see below), the Drug Attitude Inventory short form (DAI-10), ${ }^{14)}$ and the Schedule for Assessment of Insight (SAI-J). ${ }^{15)}$ The latter questionnaire was used to determine the possible risk of the patients giving mistaken answers due to cognitive dysfunction.

\section{Patient Questionnaire}

I. Evaluation of clozapine side effects and satisfaction levels

PQ1: How do you feel about the efficacy of clozapine treatment? (Efficacy)

PQ2: How do you feel about the safety of clozapine treatment? (Safety)

PQ3: How satisfied are you with the clozapine treatment you have received? (Satisfaction)

PQ4: Please list any problems associated with clozapine treatment (Problems 1)
(1) Agranulocytosis
(2) Cardiac issues
(3) Blood monitoring
(4) Diabetes mellitus
(5) Weight gain
(6) Drowsiness
(7) Hypersalivation
(8) Rebound psychosis
(9) Dizziness
(10) Nausea
(11) Constipation
(12) Other

PQ5: Do any of the problems associated with clozapine treatment make you anxious? (Problems 2)
(1) Agranulocytosis
(2) Cardiac issues
(3) Blood monitoring
(4) Diabetes mellitus
(5) Weight gain
(6) Sedation
(7) Hypersalivation
(8) Rebound psychosis
(9) Dizziness
(10) Nausea
(11) Constipation
(12) Other

PQ6: Do you want to continue clozapine treatment? (Necessity)

We categorized the questions into the following 6 themes; PQ1: efficacy, PQ2: safety, PQ3: satisfaction, PQ4 and 5: problems, and PQ6: necessity. The patients responded to PQ1-3 and PQ6 by selecting one of five answers. Multiple answers were allowed for PQ4 and 5.

II. Evaluation of adherence

Pharmacists conducted the DAI-10 to evaluate drug adherence.

III. Evaluation of insight into disease

Pharmacists conducted the SAI-J.

Questionnaire for the healthcare professionals (HPQ)

HPQ 1: How do you feel about the efficacy of clozapine 
treatment? (Efficacy)

HPQ2: How do you feel about the safety of clozapine treatment? (Safety)

HPQ3: Do you think that the patient is satisfied with their clozapine treatment? (Satisfaction)

HPQ4: What do you think are the problems associated with clozapine treatment? (Problems 1)
$\begin{array}{ll}\text { (1) Agranulocytosis (2) Cardiac issues } & \end{array}$
(3) Blood monitoring
(4) Diabetes mellitus
(5) Weight gain
(6) Sedation
(7) Hypersalivation
(8) Rebound psychosis
(9) Dizziness
(10) Nausea
(11) Constipation
(12) Other

HPQ5: What aspects of clozapine treatment is the patient anxious about? (Problems 2)
(1) Agranulocytosis (2) Cardiac issues
(3) Blood monitoring
(4) Diabetes mellitus
(5) Weight gain
(6) Sedation
(7) Hypersalivation
(8) Rebound psychosis
(11) Constipation
(9) Dizziness
(10) Nausea
(12) Other

HPQ6: Is clozapine treatment required? (Necessity)

We categorized the questions into the following 6 themes: HPQ1: efficacy, HPQ2: safety, HPQ3: satisfaction, HPQ4 and 5: problems, and HPQ6: necessity. The healthcare professionals responded to HPQ 1-3 and HPQ6 by selecting one of five answers. Multiple answers were allowed for HPQ4 and 5.

\section{Correlation studies}

We evaluated the correlations among the patients' DAI-10, SAI-J, efficacy (PQ1), safety (PQ2), satisfaction (PQ3), problems 1 (PQ4), problems 2 (PQ5), problems 3 (the sum of the scores for PQ4 and 5: We have defined that we have summed up two scores to evaluate both of present problems PQ4 and future anxious PQ5 of patient, and necessity (PQ6) scores. The responses to the PQ/HPQ were scored as follows. (H)PQ1: extremely ineffective, 1 point; ineffective, 2 points; neither agree nor disagree, 3 points; effective, 4 points; extremely effective, 5 points/ (H)PQ2: extremely unsafe, 1 point; unsafe, 2 points; neither agree nor disagree, 3 points; safe, 4 points; extremely safe, 5 points/ (H)PQ3: extremely dissatisfied, 1 point; dissatisfied, 2 points; neither agree nor disagree, 3 points; satisfied, 4 points; extremely satisfied, 5 points/ (H)PQ6: strongly disagree, 1 point; slightly disagree, 2 points; neither agree nor disagree, 3 points; slightly agree, 4 points; strongly agree, 5 points. For (H)PQ4 and 5, 1 point was awarded for each item chosen, and the total score was calculated.

\section{Statistical Analysis}

During comparisons of the main demographic and clin- ical characteristics between the groups, the paired Student's $t$-test was used to analyze continuous variables, and the chi-square test was used to analyze categorical variables. The data are presented in the form of mean \pm standard deviation values. Secondly, we used the Mann-Whitney $U$-test to compare questionnaire items (PQ1-3, PQ6, HPQ1-3, and 6). Thirdly, we used the Pearson's product-moment correlation coefficient to evaluate the relationships among the patients' DAI-10, SAI-J, PQ1, PQ2, PQ3, PQ4, PQ5, PQ4+PQ5, and PQ6 scores. $p$-values less than 0.05 were considered significant. All statistical analyses were performed using the IBM SPSS Statistics software package for Windows (version 22; IBM Co., Armonk, NY, USA).

\section{RESULTS}

\section{Background}

The background data for the patients, healthcare professionals, and the experienced/inexperienced groups are shown in Tables 1 and 2, respectively.

\section{Patients}

One hundred patients were enrolled in this study (mean age, $44.9 \pm 12.3$ years; males, $53.0 \%$; females, $47.0 \%$ ), and $68.0 \%$ of them were inpatients. The mean clozapine dosage was $381.8 \pm 148.7 \mathrm{mg}$, and the mean duration of clozapine treatment was $16.1 \pm 12.1$ months (Table 1). The mean DAI-10 score was $2.6 \pm 4.9$, and the mean SAI-J score was 10.4 \pm 3.7 (Table 1).

\section{Healthcare professionals}

One hundred and four healthcare professionals, including 54 physicians and 50 pharmacists, were enrolled in

Table 1. Patient background data $(n=100)$

\begin{tabular}{|c|c|}
\hline Variable & Data \\
\hline Age $(y r)$ & $44.9 \pm 12.3$ \\
\hline \multicolumn{2}{|l|}{ Sex } \\
\hline Male & $53(53.0)$ \\
\hline Female & $47(47.0)$ \\
\hline Clozapine dosage (mg) & $381.8 \pm 148.7$ \\
\hline Clozapine duration (mo) & $16.1 \pm 12.1$ \\
\hline Inpatients & $68(68.0)$ \\
\hline Outpatients & $32(32.0)$ \\
\hline DAI-10 score & $2.6 \pm 4.9$ \\
\hline SAI-J score & $10.4 \pm 3.7$ \\
\hline
\end{tabular}

Values are presented as mean \pm standard deviation or number (\%). DAI-10, Drug Attitude Inventory short form; SAI-J, Schedule for Assessment of Insight. 
this study. They had been working in psychiatry for a mean period of $9.8 \pm 7.8$ years (physicians: $9.2 \pm 7.4$ years, pharmacists: $10.4 \pm 7.6$ years; $p=0.293)$. Fifty-seven $(54.8 \%)$ of the healthcare professionals had experience of administering clozapine treatment (physicians: 35, 61.4\%, pharmacists: $22,38.6 \%$; $p=0.048$ ). The mean number of patients that the "experienced" staff had treated with clozapine was $7.5 \pm 17.0$ (physicians: $5.9 \pm 13.9$, pharmacists: 9.3 $\pm 19.8 ; p=0.331$ ) (Table 2).

Healthcare professionals (experienced vs. inexperienced)

The 104 healthcare professionals included 57 healthcare professionals with experience of administering clozapine and 47 healthcare professionals with no experience of administering clozapine. The staff in the "experienced" group had been working in psychiatry for $9.9 \pm 6.9$ years and had treated $12.4 \pm 18.5$ patients with clozapine ( $p=$ 0.666). The staff in the "inexperienced" group had been working in psychiatry for $13.7 \pm 21.3$ years and had never treated anyone with clozapine.

\section{Questionnaire Results}

The questionnaire results for the patients and healthcare professionals, and the experienced/inexperienced staff are shown in Table 3, respectively.

\section{Patients vs. healthcare professionals}

Sixty-six (66.0\%) patients answered "extremely effec-

Table 2. Healthcare professionals data $(n=104)$

\begin{tabular}{lccc}
\hline \multicolumn{1}{c}{ Variable } & All $(n=104)$ & Physicians $(n=54)$ & Pharmacists $(n=50)$ \\
\hline $\begin{array}{l}\text { Duration of career in psychiatry }(y r) \\
\text { Experience of clozapine treatment }\end{array}$ & $9.8 \pm 7.8$ & $9.2 \pm 7.4$ & $10.4 \pm 7.6$ \\
Experienced & $57(54.8)$ & $35(61.4)$ & $0.293^{*}$ \\
Inexperienced & $47(45.2)$ & $19(40.4)$ & $22(38.6)$ \\
Patients treated with clozapine & $7.5 \pm 17.0$ & $5.9 \pm 13.9$ & $9.048^{\dagger}$ \\
\hline
\end{tabular}

Values are presented as mean \pm standard deviation or number (\%).

*Student's t-test, ${ }^{+}$chi-square test.

Table 3. Questionnaire results regarding efficacy, safety, satisfaction and necessity

\begin{tabular}{|c|c|c|c|c|c|c|c|}
\hline & $\begin{array}{l}\text { Patients } \\
(n=100)\end{array}$ & $\begin{array}{c}\text { Healthcare } \\
\text { professionals }(n=104)\end{array}$ & $p$ value* & $\begin{array}{l}\text { Experienced } \\
\qquad(n=57)\end{array}$ & $p$ value* & $\begin{array}{l}\text { Inexperienced } \\
(n=47)\end{array}$ & $p$ value* \\
\hline Efficacy (PQ1, HPQ1) & & & $0.736^{\dagger}$ & & $0.483^{\ddagger}$ & & $0.833^{\S}$ \\
\hline Extremely effective & $28(28.0)$ & $20(19.2)$ & & $13(22.8)$ & & $7(14.9)$ & \\
\hline Effective & $38(38.0)$ & $57(54.8)$ & & $31(54.4)$ & & $26(55.3)$ & \\
\hline Neither agree nor disagree & $22(22.0)$ & $25(1.9)$ & & $11(19.3)$ & & $14(29.8)$ & \\
\hline Ineffective & $7(7.0)$ & $2(1.9)$ & & $2(3.5)$ & & $0(0.0)$ & \\
\hline Extremely ineffective & $5(5.0)$ & $0(0.0)$ & & $0(0.0)$ & & $0(0.0)$ & \\
\hline Safety (PQ2, HPQ2) & & & $0.000^{\dagger}$ & & $0.000^{\ddagger}$ & & $0.000^{\S}$ \\
\hline Extremely safe & $24(24.0)$ & $2(1.9)$ & & $2(3.5)$ & & $0(0.0)$ & \\
\hline Safe & $44(44.0)$ & $30(28.8)$ & & $20(35.1)$ & & $10(21.3)$ & \\
\hline Neither agree nor disagree & $23(23.0)$ & $46(44.2)$ & & $24(42.1)$ & & $22(46.8)$ & \\
\hline Unsafe & $6(6.0)$ & $23(22.1)$ & & $9(15.8)$ & & $14(29.8)$ & \\
\hline Extremely unsafe & $3(3.0)$ & $3(2.9)$ & & $2(3.5)$ & & $1(2.1)$ & \\
\hline Satisfaction (PQ3, HPQ3) & & & $0.002^{\dagger}$ & & $0.097^{\ddagger}$ & & $0.001^{\S}$ \\
\hline Extremely satisfied & $27(27.0)$ & $3(2.9)$ & & $3(5.3)$ & & $0(0.0)$ & \\
\hline Satisfied & $31(31.0)$ & $31(29.8)$ & & $22(38.6)$ & & $9(19.1)$ & \\
\hline Neither agree nor disagree & $26(26.0)$ & $65(62.5)$ & & $30(52.6)$ & & $35(74.5)$ & \\
\hline Dissatisfied & $10(10.0)$ & $5(4.8)$ & & $2(3.5)$ & & $3(6.4)$ & \\
\hline Extremely Dissatisfied & $6(6.0)$ & $0(0.0)$ & & $0(0.0)$ & & $0(0.0)$ & \\
\hline Necessity (PQ6, HPQ6) & & & $0.010^{\dagger}$ & & $0.020^{\ddagger}$ & & $0.293^{\S}$ \\
\hline Strongly agree & $32(32.0)$ & $40(38.5)$ & & $28(49.1)$ & & $12(25.5)$ & \\
\hline Slightly agree & $29(29.0)$ & $42(40.4)$ & & $19(33.3)$ & & $23(48.9)$ & \\
\hline Neither agree nor disagree & $19(19.0)$ & $21(20.2)$ & & $9(15.8)$ & & $12(25.5)$ & \\
\hline Slightly disagree & $7(7.0)$ & $0(0.0)$ & & $0(0.0)$ & & $0(0.4)$ & \\
\hline Strongly disagree & $13(13.0)$ & $1(1.0)$ & & $1(1.8)$ & & $0(0.0)$ & \\
\hline
\end{tabular}

Values are presented as number (\%)

$P Q$, patient questionnaire; $H P Q$, healthcare professionals questionnaire

*Mann-Whitney U-test; ${ }^{\dagger}$ patients vs. healthcare professionals, ${ }^{\neq}$patients vs. experienced, ${ }^{\S}$ patients vs. inexperienced. 
tive" or "effective" in response to PQ1, whereas 77 (74.0\%) healthcare professionals answered "extremely effective" or "effective" in response to HPQ1. Twelve $(12.0 \%)$ patients and $2(1.9 \%)$ healthcare professionals responded to (H)PQ1 with "ineffective" or "extremely ineffective" $(p=0.736)$. Sixty-eight patients $(68.0 \%)$ answered "extremely safe" or "safe" in response to PQ2, whereas $32(30.7 \%)$ healthcare professionals answered "extremely safe" or "safe" in response to HPQ2 ( $p=$ $0.000)$. Twenty-seven (27.0\%) and $31(31.0 \%)$ patients answered "extremely satisfied" and "satisfied", respectively, in response to PQ3. Three (2.9\%) and 31 $(29.8 \%)$ healthcare professionals answered "extremely satisfied" and "satisfied", respectively, in response to HPQ3 ( $p=0.002)$. A total of $61(61.0 \%)$ patients answered "strongly agree" or "agree a little" in response to PQ6, whereas $82(78.9 \%)$ healthcare professionals answered "strongly agree" or "agree a little" in response to HPQ6 $(p=0.010)$ (Table 3).

\section{Patients vs. experienced group}

Sixty-six $(66.0 \%)$ patients and $44(77.2 \%)$ healthcare professionals from the experienced group answered "extremely effective" or "effective" in response to (H)PQ1 $(p=0.483)$. Sixty-eight $(68.0 \%)$ patients and 22 $(38.6 \%)$ healthcare professionals from the experienced group answered "extremely safe" or "safe" in response to (H)PQ2 $(p=0.000)$. Twenty-seven $(27.0 \%)$ and $31(31.0 \%)$ patients answered "extremely satisfied" and "satisfied", respectively, in response to $\mathrm{PQ} 3$. In the experienced group, $2(3.5 \%)$ answered "extremely satisfied" and 20 $(35.1 \%)$ answered "satisfied" in response to the equivalent question $(p=0.000)$.

Thirty-two (32.0\%) and 29 (29.0\%) patients answered "strongly agree" and "slightly agree" in response to PQ6. In the experienced group, $47(82.4 \%)$ and 35 (74.4\%) healthcare professionals answered "strongly agree" and "slightly agree", respectively, in response to HPQ6 $(p=0.020)$ (Table 3).

\section{Patients vs. inexperienced group}

Sixty-six $(66.0 \%)$ patients and $33(70.2 \%)$ healthcare professionals from the inexperienced group answered "extremely effective" or "effective" in response to (H)PQ1 $(p=0.833)$. Sixty-eight $(68.0 \%)$ patients and $10(21.3 \%)$ healthcare professionals from the inexperienced group answered "extremely safe" or "safe" in response to (H)PQ2 $(p=0.000)$. Twenty-seven $(27.0 \%)$ and $31(31.0 \%)$ patients answered "extremely satisfied" and "satisfied", re- spectively, in response to PQ3. In the inexperienced group, none of the healthcare professionals answered "extremely satisfied", and 9 (19.1\%) answered "satisfied" in response to the equivalent question ( $p=0.001$ ).

Thirty-two (32.0\%) and 29 (29.0\%) patients answered "strongly agree" and "slightly agree", respectively, in response to PQ6 (Table 3). In the inexperienced group, 12 $(25.5 \%)$ and $23(48.9 \%)$ of the healthcare professionals answered "strongly agree" and "slightly agree", respectively, in response to HPQ6 ( $p=0.293$ ) (Table 3).

\section{Results regarding clozapine-related problems}

The questionnaire results for PQ4, PHQ4, PQ5, and PHQ5 are shown in Table 4.

PQ4: The most common response to PQ4 was hypersalivation $(76,76.0 \%)$, closely followed by drowsiness (51, 51.0\%). In addition, 40 patients $(41.0 \%)$ listed constipation as a problem (Table 4).

$P Q 5$ : The clozapine-related problems that made the patients most anxious were hypersalivation $(63,63.0 \%)$, drowsiness $(51,51.0 \%)$, and rebound psychosis $(47,47.0 \%)$ (Table 4).

HPQ4: The most common responses to HPQ4 were as follows: agranulocytosis $(98,94.2 \%)$, blood monitoring $(65,62.5 \%)$, cardiac issues $(49,47.1 \%)$, and diabetes mellitus $(49,47.1 \%)$ (Table 4$)$.

HPQ5: The most common responses to HPQ5 were as follows: hypersalivation $(54,51.9 \%)$, drowsiness (43, $41.3 \%$ ), and blood monitoring (42, 40.4\%).

The most common responses to HPQ4 in the experienced group were as follows: agranulocytosis (56, 98.2\%), blood monitoring (39, 68.4\%), and hypersalivation $(31,54.4 \%)$. The most common responses to HPQ5 in the experienced group were as follows: hypersalivation (38, 66.7\%), drowsiness, and blood monitoring (28, $49.1 \%$ ). The most common responses to HPQ4 in the inexperienced group were as follows; agranulocytosis (42, $89.4 \%$ ), blood monitoring $(26,55.3 \%)$, and diabetes mellitus $(23,48.9 \%)$. The most common responses to HPQ5 in the inexperienced group were as follows; agranulocytosis $(23,48.9 \%)$, weight gain $(18,38.3 \%)$, and hypersalivation $(16,34.0 \%)$ (Table 4).

\section{Correlation Studies}

The results of the correlation studies are shown in Table 5.

The correlation coefficients $(\mathrm{R})$ for the relationships between the patients' DAI-10 scores and their SAI-J, efficacy (PQ1), safety (PQ2), satisfaction (PQ3), problems 1 (PQ4), problems 2 (PQ5), problems 3 (the sum of the 


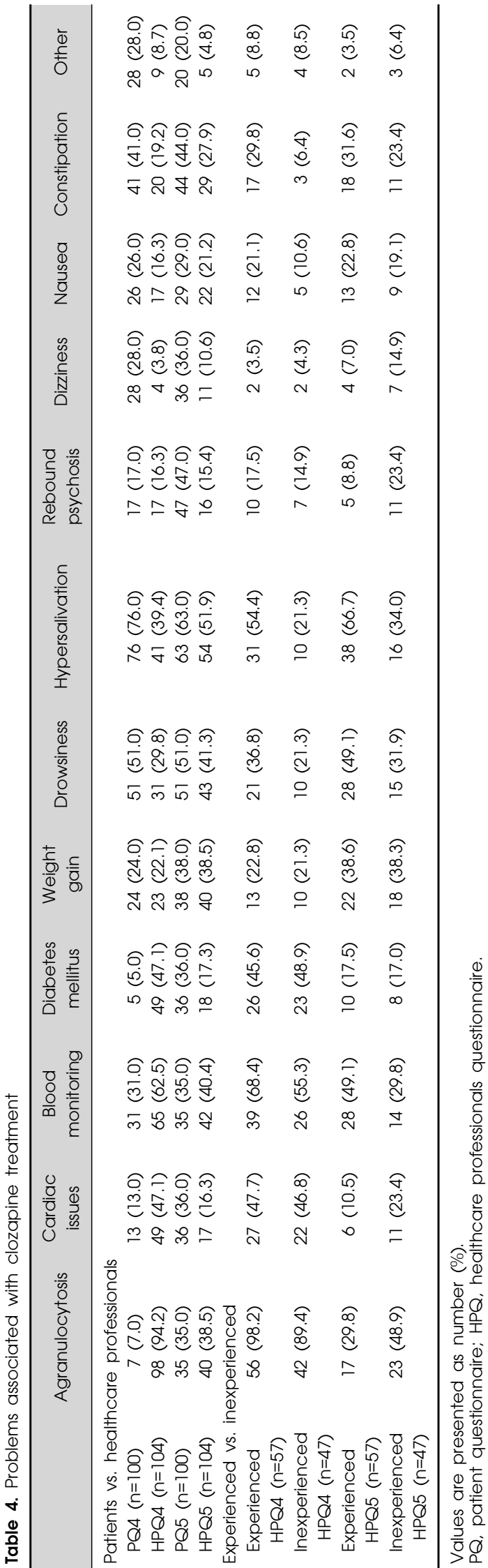

scores for PQ4 and 5), and necessity (PQ6) scores were $0.312,0.594,0.522,0.696,-0.390,-0.399,-0.433$, and 0.642 , respectively. The correlation coefficients (R) for the relationships between the patients' SAI-J scores and their efficacy, safety, satisfaction, problems 1, problems 2 , problems 3 , and necessity scores were 0.319 , $0.192,0.420,0.023,0.141,0.100$, and 0.435 , respectively. The correlation coefficients (R) for the relationships between the patients' efficacy scores and their safety, satisfaction, problems 1 , problems 2 , problems 3 , and necessity scores were $0.460,0.706,-0.129,-0.199,-0.186$, and 0.500 , respectively. The correlation coefficients (R) for the relationships between the patients' safety scores and their satisfaction, problems 1 , problems 2 , problems 3 , and necessity scores were $0.550,-0.166,-0.199$, 0.203 , and 0.507 ), respectively. The correlation coefficients (R) for the relationships between the patients' satisfaction scores and their problems 1 , problems 2 , problems 3 , and necessity scores were $-0.258,-0.237$, 0.269 , and 0.627 , respectively. The correlation coefficients (R) for the relationships between the patients' problems 1 scores and their problems 2 , problems 3 , and necessity scores were $0.657,0.877$, and -0.282 , respectively. The correlation coefficients $(\mathrm{R})$ for the relationships between the patients' problems 2 scores and their problems 3 and necessity scores were 0.939 and 0.248 , respectively. Finally, the correlation coefficient (R) for the relationship between the patients' problems 3 and necessity scores was -0.287 (Table 5).

\section{DISCUSSION}

Clozapine was first introduced as a treatment for schizophrenia in Austria in 1969, but it stopped being used in 1975 after a report from Finland found that it caused agranulocytosis. However, in 1988 further studies demonstrated that clozapine is effective against refractory schizophrenia. As a result, clozapine was approved for use as a treatment for refractory schizophrenia in 1989 after the establishment of the Clozapine Patient Monitoring Service (CPMS) by the United States Food and Drug Administration. The CPMS developed strict leukocyte and neutrophil count standards for patients receiving clozapine, which resulted in reductions in the frequencies of agranulocytosis and mortality. Whilst the establishment of the CPMS increased the safety of clozapine treatment, it also led to negative views about the drug among healthcare professionals. This phenomenon might partly explain the discrepancy between the views of patients and health- 
Table 5. Correlation study results

\begin{tabular}{|c|c|c|}
\hline \multicolumn{2}{|r|}{ Item of variable } & \multirow{2}{*}{$\begin{array}{c}\text { The correlation } \\
\text { coefficients (R) } \\
0.312\end{array}$} \\
\hline DAl-10 & SAl-J & \\
\hline & Efficacy (PQ1) & 0.594 \\
\hline & Safety (PQ2) & 0.522 \\
\hline & Satisfaction (PQ3) & 0.696 \\
\hline & Problems 1 (PQ4) & -0.390 \\
\hline & Problems 2 (PQ5) & -0.399 \\
\hline & Problems 3 (PQ4 and 5) & -0.433 \\
\hline & Necessity (PQ6) & 0.642 \\
\hline \multirow[t]{7}{*}{ SAl-J } & Efficacy (PQ1) & 0.319 \\
\hline & Safety (PQ2) & 0.192 \\
\hline & Satisfaction (PQ3) & 0.420 \\
\hline & Problems 1 (PQ4) & 0.023 \\
\hline & Problems 2 (PQ5) & 0.141 \\
\hline & Problems 3 (PQ4 and 5) & 0.100 \\
\hline & Necessity (PQ6) & 0.435 \\
\hline \multirow[t]{6}{*}{ Efficacy (PQ1) } & Safety (PQ2) & 0.460 \\
\hline & Satisfaction (PQ3) & 0.706 \\
\hline & Problems 1 (PQ4) & -0.129 \\
\hline & Problems 2 (PQ5) & -0.199 \\
\hline & Problems 3 (PQ4 and 5) & -0.186 \\
\hline & Necessity (PQ6) & 0.500 \\
\hline \multirow[t]{5}{*}{ Safety (PQ2) } & Satisfaction (PQ3) & 0.550 \\
\hline & Problems 1 (PQ4) & -0.166 \\
\hline & Problems 2 (PQ5) & -0.199 \\
\hline & Problems 3 (PQ4 and 5) & -0.203 \\
\hline & Necessity (PQ6) & 0.507 \\
\hline \multirow{4}{*}{$\begin{array}{l}\text { Satisfaction } \\
\text { (PQ3) }\end{array}$} & Problems 1 (PQ4) & -0.258 \\
\hline & Problems 2 (PQ5) & -0.237 \\
\hline & Problems 3 (PQ4 and 5) & -0.269 \\
\hline & Necessity (PQ6) & 0.627 \\
\hline \multirow{3}{*}{$\begin{array}{l}\text { Problems } 1 \\
\text { (PQ4) }\end{array}$} & Problems 2 (PQ5) & 0.657 \\
\hline & Problems 3 (PQ4 and 5) & 0.877 \\
\hline & Necessity (PQ6) & -0.282 \\
\hline \multirow{2}{*}{$\begin{array}{l}\text { Problems } 2 \\
\text { (PQ5) }\end{array}$} & Problems 3 (PQ4 and 5) & 0.939 \\
\hline & Necessity (PQ6) & -0.248 \\
\hline $\begin{array}{l}\text { Problems } 3 \\
(P Q 4 \text { and 5) }\end{array}$ & Necessity (PQ6) & -0.287 \\
\hline
\end{tabular}

DAI-10, Drug Attitude Inventory short form; SAI-J, Schedule for Assessment of Insight; $P Q$, patient questionnaire.

care professionals regarding clozapine treatment. Therefore, we decided to research the discrepancy between patient feedback regarding clozapine treatment and the views of healthcare professionals about the same topic.

This study involved 100 patients and 104 healthcare professionals. The efficacy scores of the patients and healthcare professionals did not differ significantly. In total, $64.0 \%$ of patients, $77.2 \%$ of the experienced healthcare professionals, and $70.2 \%$ of the inexperienced healthcare professionals considered clozapine to be "extremely effective" or "effective". Thus, all three groups realized that clozapine is effective. This result agrees with the findings of a previous study. ${ }^{16)}$ Clozapine was reported to be effective in previous study, ${ }^{1)}$ and even the inex- perienced staff seemed to be aware of this. Regarding safety, $24 \%$ of the patients, $3.5 \%$ of the experienced healthcare professionals, and none of the inexperienced healthcare professionals considered clozapine treatment to be "extremely safe". The scores for this question differed significantly between the patients, experienced healthcare professionals, and the inexperienced healthcare professionals. None of the patients developed serious side effects such as agranulocytosis, which explains why they considered clozapine treatment to be highly safe. However, healthcare professionals understand that no matter how effective clozapine is, it is associated with serious risks. Regarding the subjects' satisfaction scores, a significant difference was detected between the scores of the patients and those of the inexperienced healthcare professionals, but no such difference was observed between the patients' scores and those of the experienced healthcare professionals. As for the question regarding the necessity of clozapine treatment, the scores of the patients and experienced healthcare professionals differed significantly, but no such difference was detected between the patients' scores and those of the inexperienced healthcare professionals. The experienced healthcare professionals correctly understood the risk of side effects during clozapine treatment and the satisfaction levels of clozapine patients. Furthermore, they were more firmly of the view that clozapine treatment was necessary than the patients. The inexperienced healthcare professionals agreed that clozapine is effective and necessary. However, they considered clozapine to be unsafe, which led to them to take the view that clozapine treatment results in low patient satisfaction. This view might be an obstacle to the propagation of clozapine treatment in Japan and prevent patients from benefiting from such treatment.

The most common problem experienced by the patients was hypersalivation, followed by drowsiness and constipation. As for the clozapine treatment-related issues that made the patients most anxious, hypersalivation was the most frequently identified problem, followed by drowsiness and rebound psychosis. Thus, hypersalivation was the most common response to both questions. Hypersalivation occurs in $31.0-97.4 \%$ of patients treated with clozapine. ${ }^{17,18)}$ It usually develops early in the course of treatment. Previous reports have indicated that scopolamine $^{19)}$ and scopolamine butylbromide ${ }^{20)}$ (anticholinergic agents) are effective against hypersalivation. However, the treatment of hypersalivation remains incompletely understood. So, we need to find a fast-acting solution to this problem. Interestingly, many of the patients were con- 
cerned about rebound psychosis. Despite this, it is clear that the patients were fully aware of the importance of clozapine treatment from their answers. In contrast to the patients' answers, the most common response of the healthcare professionals regarding the problems associated with clozapine treatment was agranulocytosis, followed by blood monitoring. During clozapine treatment, it is obviously extremely important to prevent death from agranulocytosis. Therefore, it is good that the risk of agranulocytosis during clozapine treatment is widely known. However, the healthcare professionals' knowledge about the risk of agranulocytosis during clozapine treatment seemed to have a very big impact on their views about the drug, and the healthcare professionals were also concerned about diabetes mellitus and cardiac issues. However, hypersalivation, drowsiness, and constipation were the most frequently listed clozapine treatment-associated problems by the patients. It is important to recognize these differences.

In the correlation analysis, several items were found to be correlated with each other. The DAI-10 score was positively correlated with satisfaction and necessity, and was negatively correlated with problems. These results suggest that the numerous problems associated with clozapine treatment have the potential to decrease levels of adherence. Thus, clozapine treatment could place a heavy burden on patients.

We acknowledge that this study has some limitations. Firstly, this study targeted common side effects, although it had the potential to detect rare side effects. Secondly, the clozapine dosage and the duration of treatment differed greatly among the patients.

This study showed that there is a big gap between the views of experienced and inexperienced healthcare professionals regarding clozapine treatment. Despite the low adherence seen among clozapine-treated patients, which can be explained in part by the side effects of the drug, the current clinical practice surrounding the use of clozapine is insufficient. In this context, it is our wish to spread the usage of clozapine. Clozapine treatment always results in side effects, but the drug's effectiveness makes it a very important tool for treating schizophrenia. In other words, higher risk treatment should not necessarily be equated with useless treatment. The appropriate management of clozapine's side effects would improve adherence, and therefore, might help clozapine-treated patients return to employment. In addition it would help to enable patients to derive the maximum benefit from clozapine treatment.

\section{REFERENCES}

1. Conley RR, Buchanan RW. Evaluation of treatment-resistant schizophrenia. Schizophr Bull 1997;23:663-674.

2. Kane J, Honigfeld G, Singer J, Meltzer H. Clozapine for the treatment-resistant schizophrenic. A double-blind comparison with chlorpromazine. Arch Gen Psychiatry 1988; 45:789-796.

3. Alvir JM, Lieberman JA, Safferman AZ, Schwimmer JL, Schaaf JA. Clozapine-induced agranulocytosis. Incidence and risk factors in the United States. $N$ Engl $J$ Med 1993;329:162-167.

4. Alvir JM, Lieberman JA. Agranulocytosis: incidence and risk factors. J Clin Psychiatry 1994;55 Suppl B:137-138.

5. Davydov L, Botts SR. Clozapine-induced hypersalivation. Ann Pharmacother 2000;34:662-665.

6. Stroup TS, Gerhard T, Crystal S, Huang C, Olfson M. Comparative effectiveness of clozapine and standard antipsychotic treatment in adults with schizophrenia. Am J Psychiatry 2016;173:166-173.

7. Nielsen J, Dahm M, Lublin H, Taylor D. Psychiatrists' attitude towards and knowledge of clozapine treatment. $J$ Psychopharmacol 2010;24:965-971.

8. Hodge K, Jespersen S. Side-effects and treatment with clozapine: a comparison between the views of consumers and their clinicians. Int J Ment Health Nurs 2008;17:2-8.

9. Day JC, Wood G, Dewey M, Bentall RP. A self-rating scale for measuring neuroleptic side-effects. Validation in a group of schizophrenic patients. Br J Psychiatry 1995;166: 650-653.

10. Howes OD, Vergunst F, Gee S, McGuire P, Kapur S, Taylor D. Adherence to treatment guidelines in clinical practice: study of antipsychotic treatment prior to clozapine initiation. Br J Psychiatry 2012;201:481-485.

11. Valenstein M, Copeland LA, Blow FC, McCarthy JF, Zeber JE, Gillon L, et al. Pharmacy data identify poorly adherent patients with schizophrenia at increased risk for admission. Med Care 2002;40:630-639.

12. Kane JM. Management strategies for the treatment of schizophrenia. J Clin Psychiatry 1999;60 Suppl 12:13-17.

13. Sweileh WM, Ihbesheh MS, Jarar IS, Sawalha AF, Abu Taha AS, Zyoud SH, et al. Antipsychotic medication adherence and satisfaction among Palestinian people with schizophrenia. Curr Clin Pharmacol 2012;7:49-55.

14. Hogan TP, Awad AG, Eastwood R. A self-report scale predictive of drug compliance in schizophrenics: reliability and discriminative validity. Psychol Med 1983;13: 177-183.

15. David AS. Insight and psychosis. Br J Psychiatry 1990; 156:798-808.

16. Angermeyer MC, Löffler W, Müller P, Schulze B, Priebe S. Patients' and relatives' assessment of clozapine treatment. Psychol Med 2001;31:509-517.

17. Zeng XP, Le F, Richelson E. Muscarinic $m 4$ receptor activation by some atypical antipsychotic drugs. Eur J Pharmacol 1997;321:349-354.

18. Kishi T, Fujita K, Furukawa O, Suzuki T, Moriwaki M, Nitta M, et al. Efficacy and tolerability of clozapine in Japanese patients with treatment-resistant schizophrenia: results from a 12-week, flexible dose study using raters masked to antipsychotic choice. Asian J Psychiatr 2013;6: 200-207.

19. McKane JP, Hall C, Akram G. Hyoscine patches in cloza- 
294 I Takeuchi, et al.

pine-induced hypersalivation. Psychiatr Bull 2001;25:277.

20. Takeuchi I, Suzuki T, Kishi T, Kanamori D, Hanya M, Uno

$\mathrm{J}$, et al. Effect of scopolamine butylbromide on clozapine- induced hypersalivation in schizophrenic patients: A case series. Clin Psychopharmacol Neurosci 2015;13:109-112. 